\title{
Global Strategy for the Diagnosis, Management and Prevention of COPD: 2003 update
}

\author{
L.M. Fabbri*, S.S. Hurd", for the GOLD Scientific Committee
}

The Global Initiative for Chronic Obstructive Lung Disease (GOLD) programme was initiated in January 1997 to increase awareness of chronic obstructive pulmonary disease (COPD) and to decrease morbidity and mortality from this chronic lung disorder. One strategy to help achieve the objectives of the GOLD programme is to provide healthcare workers, healthcare authorities and the general public with state-of-the-art information about COPD and specific recommendations on the most appropriate management and prevention strategies.

The GOLD Workshop Report, Global Strategy for the Diagnosis, Management and Prevention of COPD [1] was published in April 2001. It was prepared by a panel of experts nominated by the National Heart, Lung and Blood Institute (NHLBI), National Institutes of Health and the World Health Organization with the aim of providing the best validated current concepts of COPD pathogenesis and the best available evidence on the most appropriate management and prevention strategies. In an effort to keep the GOLD Workshop Report as up to date as possible, GOLD assembled a Scientific Committee whose aim was to review clinical research that has an impact on COPD management. The initial review included publications that were published in June 2000 (approximately the time of completion of the 2001 report) through to March 2003. The results of the first 2 yrs of activity were posted on the GOLD website (www.goldcopd. com) in July 2003 [2]. Each year, a new update report will be posted. The GOLD Scientific Committee will also prepare a revision of the entire GOLD Workshop Report approximately every 5 yrs. The process for the first complete revision (to appear in 2006) will be developed in the autumn of 2003.

\section{Method}

The process included a PubMed search using search fields established by the GOLD Scientific Committee: 1) COPD OR chronic bronchitis OR emphysema, All Fields, All Adult, 19+ yrs, only items with abstracts, Clinical Trial, Human, sorted by Authors; and 2) COPD OR chronic bronchitis OR emphysema AND systematic, All fields, All adult, 19+ years, only items with abstracts, Human, sort by Author. In addition, publications in peer-review journals not captured by PubMed could be submitted to individual members of the Committee, providing an abstract and the full paper were submitted in (or translated into) English.

*Dept Respiratory Medicine, University of Medena \& Reggio, Modena, Italy. ${ }^{\#}$ Scientific Director, Global Initiative on Obstructive Lung Disease (GOLD), Gaithersburg, MD, USA.

Correspondence: L.M. Fabbri, Dept Respiratory Medicine, University of Medena \& Reggio, Via del Pozzo, 71, 41100 Modena, Italy. Fax: 39 0594224231. E-mail: fabbri.leoardo@unimo.it
All members of the GOLD Scientific Committee received a summary of citations and all of the abstracts. Each abstract was assigned to two members (members were not assigned to a paper where they appeared as an author), although all members were given the opportunity to provide an opinion on any abstract. They were then asked to evaluate the abstract or, if they chose, the full publication, by answering specific written questions from a short questionnaire, and to indicate if the scientific data presented in the paper made an impact on recommendations in the GOLD Workshop Report. If so, the member was asked to specifically identify modifications that should be made. The entire GOLD Scientific Committee met on a regular basis to discuss each individual publication that was considered, by at least one member, to have an impact on the report, and to reach a consensus on the changes in the report. Disagreements were resolved by vote.

\section{Level of evidence}

Guidelines based solely on a consensus of expert opinion or on unsystematic literature surveys may not always reflect current medical knowledge and are liable to bias [3, 4]. Therefore, with the aim of identifying and summarising evidence of the effectiveness of interventions, to allow the generalisability and consistency of research findings to be assessed and to allow data inconsistency to be explored, the GOLD Scientific Committee conducted a review of the evidence both in the preparation of the 2001 GOLD Workshop Report and in its update process.

The Scientific Committee reviewed a variety of evidence tables and chose to assign levels of evidence to management recommendations using the system developed by the NHLBI. As with most adopted systems, this system assigns the strongest level of evidence (evidence A) for supporting recommendations to randomised clinical trials. This system also includes post-hoc or subgroup analysis of randomised clinical trials and meta-analysis of randomised clinical trials as evidence, although these types of papers are assigned a lower level of evidence (e.g. evidence B).

During the updating process, there has been considerable debate on criteria to assign evidence, in particular: 1) the methodology to assess the validity of papers identified as potential sources of evidence; and 2) meta-analyses and systematic reviews. Apart from specific questions reported in the questionnaire of evaluating individual publications, the scientific evaluation was left to the individual member. However, as the assessment process involves a degree of subjective judgment, each study was evaluated independently by at least two members of the Committee and then by the entire Committee. With regard to the value assigned to meta-analyses and systematic reviews, the GOLD Scientific Committee decided that these publications may be cited as references, but that a level of evidence could not be assigned to a recommendation based on a meta-analysis and/or systematic 
review alone. This decision was taken because the Committee considered: 1) that their responsibility was to assess the validity of original studies, i.e. of the individual randomised clinical trials, rather than to rely on the evaluation of the authors of meta-analyses or systematic reviews; and 2) that meta-analysis or post-hoc analysis of pooled randomised clinical trials, individually insufficient to provide a definite statistically significant answer to the main question, may be used to generate an hypothesis for prospective randomised clinical trials rather provide scientific evidence.

\section{The 2003 update of the April 2001 GOLD Workshop Report}

The GOLD Scientific Committee reviewed publications $(n=241)$ that appeared between June 2000 and March 2003. There were a total of 36 papers were identified that had an impact on the GOLD report, either by confirming, i.e. adding or replacing an existing reference, or modifying, i.e. changing the text or introducing a concept that required a new recommendation to the report, original data. The most important modifications introduced to the management section of the report were as follows. 1) The position of long- and shortacting bronchodilators, including the introduction of the new long-acting anticholinergic tiotropium. 2) The position of inhaled glucocorticosteroids and the combination of inhaled long-acting $\beta_{2}$-agonists/glucocorticosteroids. 3) Evidence related to length of pulmonary rehabilitation programmes. 4) Home versus hospital care for COPD exacerbations.

Because of difficulties encountered using the 2001 GOLD classification by severity in the dissemination process and in line with the recommendations that are being proposed by the COPD Guidelines Committee (nominated jointly by the European Respiratory Society and the American Thoracic Society), the classification was maintained but the stages of severity were renamed as $\mathrm{I}=$ mild, $\mathrm{II}=$ moderate, $\mathrm{III}=$ severe and IV=very severe, instead of stages Mild (I), IIA, IIB and III, respectively. Stage 0 was not changed.

The 2001 GOLD Workshop Report included the recommendation to use regular treatment with bronchodilators for moderate-to-severe COPD, stating that long-acting bronchodilators were more convenient than short-acting bronchodilators. Based on publications that appeared after June 2000, the 2003 GOLD Update recommends, for moderate-to-very severe COPD, use of regular treatment with long-acting bronchodilators, including tiotropium, rather than shortacting bronchodilators, with an evidence level A.

The 2001 GOLD Workshop Report included the recommendation to use inhaled glucocorticosteroids for patients with COPD of severity II (moderate) or more, providing they had a spirometric response to a short-term course of steroids and/or a forced expiratory volume in one second of $<50 \%$ predicted and frequent exacerbations. This recommendation was assigned evidence $\mathrm{B}$, reflecting the inconsistency of response to inhaled glucocorticosteroids reported in the literature. Based on publications appearing after June 2000, the 2003 GOLD Update recommends the use of inhaled glucocorticosteroids only in patients with COPD of severity III and frequent exacerbations, assigning to the recommendation an evidence A, reflecting the consistency of the response to inhaled glucocorticosteroids in more severe patients reported in the literature.

The 2001 GOLD Workshop Report did not include a specific recommendation for the duration of rehabilitation programmes. Based on publications appearing after June 2000 , the 2003 GOLD Update recommends a duration of $\geqslant 2$ months for rehabilitation programmes, assigning to the recommendation evidence $\mathrm{B}$, reflecting the limited number of studies available.
The 2001 GOLD Workshop Report did not include a specific recommendation for nurse-administered homecare as an alternative to hospitalisation of patients with COPD exacerbations. Based on publications appearing after June 2000, the 2003 GOLD Update suggests that nurse-administered homecare represents an effective and practical alternative to hospitalisation in selected patients with exacerbations of COPD without acidotic respiratory failure. However, because the exact criteria for home versus hospital treatment remains uncertain and may vary by healthcare setting, no level of evidence was assigned to this recommendation.

Finally, in addition to small changes and the correction of mistakes contained in the original document, the Committee identified important issues (e.g. antibiotic treatment of COPD exacerbations, step-up/down of pharmacological treatment, use of walking aids for rehabilitation, anesthesia in severe COPD patients undergoing surgery) for which the new scientific evidence reviewed was considered insufficient to change the 2001 Report, but that were judged as priority issues to be addressed in the 2004 update. Prior to release, the proposed modifications to the 2001 GOLD Workshop Report were submitted to the GOLD Executive Committee for approval. A copy of the 2003 GOLD Workshop Report Update, along with the accompanying documents (Executive Summary and Pocket Guide) and the complete list of references examined by the Committee are now available on the GOLD website.

The significant number of changes and their impact on patient care highlighted the need for continuous update both to provide the best recommendations and to improve the quality of the report. This exercise reinforced the decision of the Global Initiative for Chronic Obstructive Lung Disease Scientific Committee to regularly repeat the update process, posting an updated version of the text of the Management sections on the website each year.

\footnotetext{
Acknowledgements. Members of the GOLD Scientific Committee: L. Fabbri (Chair); P. Barnes, S. Buist, P. Calverley, Y. Fukuchi, S. Hurd, W. MacNee, R. Pauwels, K. Rabe, R. RodriguezRoisin, J. Zielinski.

Members of the GOLD World Expert Panel: R. Pauwels (Chair); N. Anthonisen, W. Bailey, P. Barnes, S. Buist, P. Calverley, T. Clark, L. Fabbri, Y. Fukuchi, L. Grouse, J. Hogg, C. Jenkins, D. Postma, K. Rabe, S. Ramsey, S. Rennard, R. Rodriguez-Roisin, N. Siafakas, S. Sullivan, W.C. Tan, S. DeWeerdt (editor), S. Hurd. Members of the GOLD Executive Committee: R. Pauwels (Chair); Buist, P. Calverley, B. Celli, J.L. Fabbri, Y. Fukuchi, L. Grouse, S. Hurd, C. Jenkins, C. Lenfant, T. van der Molen, E. Nizankowska-Mogilnicka, K. Rabe, R. RodriguezRoisin, N-S. Zhong.
}

\section{References}

1. Global Initiative for Chronic Obstructive Lung Disease. Global Strategy for the Diagnosis, Management and Prevention of Chronic Obstructive Pulmonary Disease. NHLBI/ WHO workshop report. Bethesda, National Heart, Lung and Blood Institute, April 2001; NIH Publication No. 2701: 1-100.

2. Global Initiative for Chronic Obstructive Lung Disease. Global Strategy for the Diagnosis, Management and Prevention of Chronic Obstructive Pulmonary Disease. NHLBI/WHO workshop report. Bethesda, National Heart, Lung and Blood Institute, April 2001; Update of the Management Sections, GOLD website (www.goldcopd.com). Date updated: July 2003.

3. Shekelle PG, Woolf SH, Eccles M, Grimshaw J. Developing guidelines. BMJ 1999; 318: 593-536. 